International Journal of Mechanical Engineering and Technology (IJMET)

Volume 11, Issue 7, July 2020, pp. 38-50, Article ID: IJMET_11_07_005

Available online at https://iaeme.com/Home/issue/IJMET?Volume=11\&Issue=7

ISSN Print: 0976-6340 and ISSN Online: 0976-6359

DOI: https://doi.org/10.34218/IJMET.11.7.2020.005

(C) IAEME Publication

\title{
HIGHER ORDER REFINED COMPUTATIONAL MODEL USING POST PROCESSING FOR THE TRANSVERSE SHEAR AND TRANSVERSE NORMAL STRESS IN COMPOSITE PLATES
}

\author{
Rohit Govind Sangwai and Niloy K. Nath \\ Department of Mechanical Engineering, JSPM's Rajarshi Shahu College of Engineering, \\ Savitribai Phule Pune University, Pune, India
}

\begin{abstract}
Many researchers presented failure analysis of multilayered plates. Some researchers used Equivalent Single Layer (ESL) for their analysis. Some researchers adopted layer wise theories for failure analysis. Accuracy in estimation of transverse stresses plays a pivotal role in such analysis. Transverse stresses at the layer interface calculated from the constitutive equation gives dis continuity of stresses. As such these stresses can be precisely forecasted by applying a technique. This Technique includes integration of the $3 D$ equilibrium equations as post processing. The principal aim of the present work is to evaluate analytically the accuracy of a higher order refined computational model with 12 unknowns in predicting the transverse shear and normal stresses. The theoretical model presented herein considers the effects of both transverse shear and normal strain/stress. This model also considers the warping of transverse cross sections. The theoretical formulations and solution method for post processing is presented in the paper. Extensive numerical results are presented and discussed. Present model with post processing could predict the transverse shear and normal stresses more accurately than the models with lower variable.
\end{abstract}

Keywords: Composite Laminates, Transverse Stresses, Higher Order Shear Deformation Theory, Analytical Method.

Cite this Article: Rohit Govind Sangwai and Niloy K. Nath, Higher Order Refined Computational Model using Post Processing for the Transverse Shear and Transverse Normal Stress in Composite Plates. International Journal of Mechanical Engineering and Technology. 11(7), 2020, pp. 38-50.

https://iaeme.com/Home/issue/IJMET?Volume $=11 \&$ Issue $=7$

\section{INTRODUCTION}

Analysis of laminated of such covered composites gets troublesome because of various aspects. Material mismatch across the laminate interfaces is one of the aspect, Bendingstretching coupling and geometric non-linear effects are other aspects. Laminated composites have low transverse shear stiffness. Therefore laminated composites having lower side to 
thickness ratio are significantly affected transverse shear deformation. Accuracy in predicting the transverse stresses are most important for predicting failure of composites. Various investigators used numerous theories for the accurate computation of the inplane and transverse stresses to be used in failure analysis. A complete review of literature up to the year 2019 is also found in the articles presented by many authors [1-7]. Some of the researchers are of the opinion that ESL can only predict global responses accurately. Deflection, natural frequency and buckling load comes under global response. These researchers are of the opinion that ESL theories fail to predict local response. Variation of stress distribution comes under local response. In their opinion layerwise theories which are computationally expensive as compared to ESL theories can only predict exact through the thickness variation of stresses. Most of the researchers used computational models with up to a maximum of five degrees of freedom in their analysis to arrive at these conclusions. Some researchers are of opposite school of thoughts [8-11]. In this paper one such higher order shear deformation theory with 12 DOF already available in the literature is considered. Analytical formulation and solution method to compute the transverse stresses by using post processing techniques not yet reported. Extensive numerical results using this model are provided. Analytical solutions for the in-plane stresses of plates using this computational model are reported in the articles by Swaminatan and Patil [13-15], Kant and Manjunatha [12]. It was shown that the results of linear stresses obtained using the model is very much closer to 3D elasticity solutions. Antisymmetric angle ply laminates are used to have more torsional rigidity viz. in fan blades. There is not much results published in the literature on the transverse stresses of antisymmetric angle ply composite plates.

\section{THEORETICAL FORMULATION\&ANALYTICAL SOLUTION}

Equivalent Single layer theory is used for the analysis. The displacement field derived from the expanded Taylor's series in terms of the thickness coordinates $\mathrm{z}$ and having 12 DOF proposed by Kant and Manjunatha [12] is considered

$$
\begin{aligned}
& \mathrm{u}(\mathrm{x}, \mathrm{y}, \mathrm{z})=\mathrm{u}_{\mathrm{o}}(\mathrm{x}, \mathrm{y})+\mathrm{z} \theta_{\mathrm{x}}(\mathrm{x}, \mathrm{y})+\mathrm{z}^{2} \mathrm{u}_{\mathrm{o}}^{*}(\mathrm{x}, \mathrm{y})+\mathrm{z}^{3} \theta_{\mathrm{x}}^{*}(\mathrm{x}, \mathrm{y}) \\
& \mathrm{v}(\mathrm{x}, \mathrm{y}, \mathrm{z})=\mathrm{v}_{\mathrm{o}}(\mathrm{x}, \mathrm{y})+\mathrm{z} \theta_{\mathrm{y}}(\mathrm{x}, \mathrm{y})+\mathrm{z}^{2} \mathrm{v}_{\mathrm{o}}^{*}(\mathrm{x}, \mathrm{y})+\mathrm{z}^{3} \theta_{\mathrm{y}}^{*}(\mathrm{x}, \mathrm{y}) \\
& w(x, y, z)=w_{o}(x, y)+z \theta_{z}(x, y)+z^{2} w_{o}^{*}(x, y)+z^{3} \theta_{z}^{*}(x, y)
\end{aligned}
$$

Where

$\mathrm{u}_{\mathrm{o}}, \mathrm{v}_{\mathrm{o}}$ and $\mathrm{w}_{\mathrm{o}}$ are the displacements on the middle plane.

$\theta_{x}, \theta_{y}$ and $\theta_{z}$ are rotations

Others are higher order terms. These terns are developed from the Taylor's series expansion.

For modeling each layer of laminate is assumed as homogeneous and orthotropic and the laminate throughout the thickness is assumed as anisotropic and heterogeneous.

The general linear strain-displacement relations are given as follows:

$$
\varepsilon_{x}=\frac{\partial u}{\partial x} ; \varepsilon_{y}=\frac{\partial v}{\partial y} ; \gamma_{x y}=\frac{\partial u}{\partial y}+\frac{\partial v}{\partial x} \varepsilon_{z}=\frac{\partial w}{\partial z} ; \gamma_{y z}=\frac{\partial v}{\partial z}+\frac{\partial w}{\partial y} ; \gamma_{x z}=\frac{\partial u}{\partial z}+\frac{\partial w}{\partial x}
$$




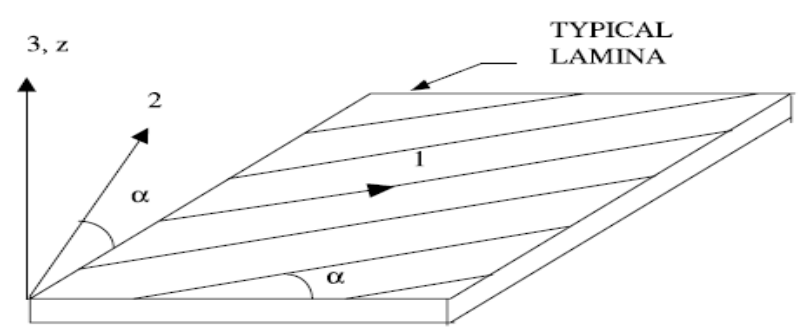

( $1,2,3$ ) - LAMINA REFERENCE AXES

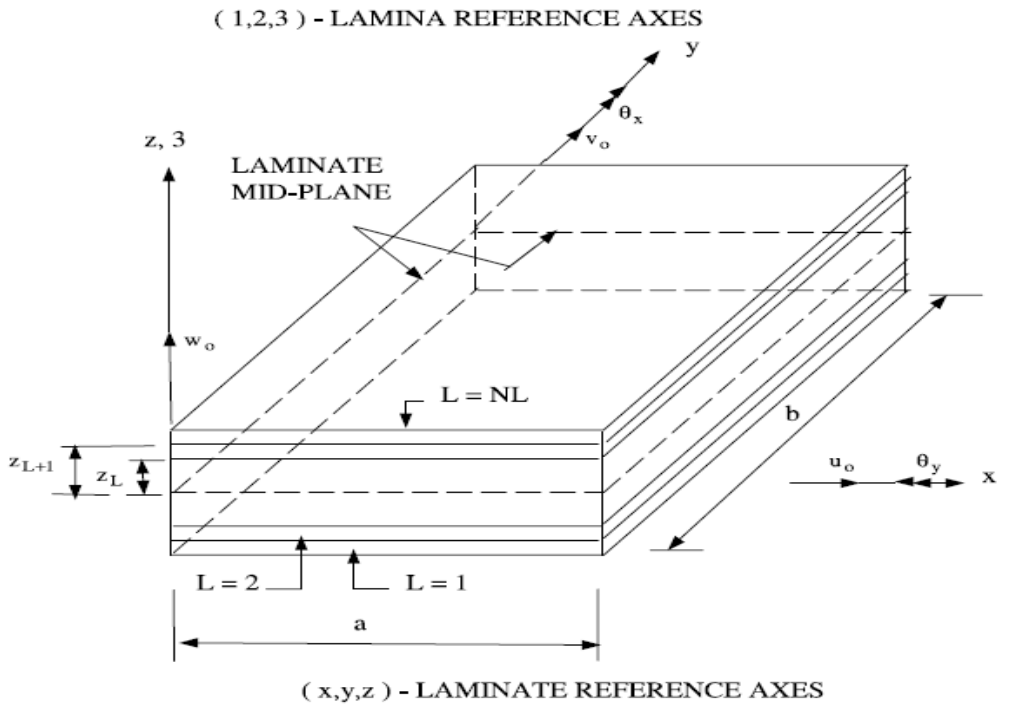

Figure 1 Laminate Geometry with positive set of Lamina/laminate reference axes, displacement components Thus,

The stress-strain relations for $\mathrm{L}^{\text {th }}$ lamina is developed. Figure 1 gives laminate geometry.

$$
\{\sigma\}^{L}=\left[Q_{i j}\right]\{\varepsilon\}^{L}
$$

where $\{\sigma\}^{L}=\left(\sigma_{x}, \sigma_{y}, \sigma_{z}, \tau_{x y}, \tau_{y z}, \tau_{x z}\right)$ are the stresses and

$\{\varepsilon\}^{L}=\left(\varepsilon_{x}, \varepsilon_{y}, \varepsilon_{z}, \gamma_{x y}, \gamma_{y z}, \gamma_{x z}\right)$ are the strains with respect to the laminate

axes.

$\left\lfloor Q_{i j}\right\rfloor_{\text {are the transformed elastic constants or the stiffness matrix with respect }}$

to the laminate axes $\mathrm{x}, \mathrm{y}, \mathrm{z}$.

Authors have independently analysed in plane stresses. But due to space restraint are not provided here. Readers may refer papers of K. Swaminathan and Patil [12-15] for detailed formulation, analysis and solution to get in in plane stresses. Afterwards Transverse stresses are calculated by applying post processing technique. This technique includes integration of $3 \mathrm{D}$ elasticity equilibrium equations. The procedure given below:

The equilibrium equations are:

$$
\frac{\partial \sigma_{\mathrm{x}}}{\partial \mathrm{x}}+\frac{\partial \tau_{\mathrm{xy}}}{\partial \mathrm{y}}+\frac{\partial \tau_{\mathrm{xz}}}{\partial \mathrm{z}}=0 ; \frac{\partial \tau_{\mathrm{xy}}}{\partial \mathrm{x}}+\frac{\partial \sigma_{\mathrm{y}}}{\partial \mathrm{y}}+\frac{\partial \tau_{\mathrm{yz}}}{\partial \mathrm{z}}=0 ; \frac{\partial \tau_{\mathrm{xz}}}{\partial \mathrm{x}}+\frac{\partial \tau_{\mathrm{yz}}}{\partial \mathrm{y}}+\frac{\partial \sigma_{\mathrm{z}}}{\partial \mathrm{z}}=0
$$

Integrating the above set of equations with respect to the thickness coordinate ' $z$ ' we get 
Higher Order Refined Computational Model using Post Processing for the Transverse Shear and Transverse Normal Stress in Composite Plates

$$
\begin{gathered}
\tau_{\mathrm{xz}}^{(\mathrm{L})}=-\int_{\mathrm{z}_{1}}^{\mathrm{z}}\left(\frac{\partial \sigma_{\mathrm{x}}^{(\mathrm{L})}}{\partial \mathrm{x}}+\frac{\partial \sigma_{\mathrm{xy}}^{(\mathrm{L})}}{\partial \mathrm{y}}\right) \mathrm{dz}+\mathrm{C}_{1}^{(\mathrm{L})}(\mathrm{x}, \mathrm{y}) \\
\tau_{\mathrm{yz}}^{(\mathrm{L})}=-\int_{\mathrm{z}_{1}}^{\mathrm{z}}\left(\frac{\partial \sigma_{\mathrm{xy}}^{(\mathrm{L})}}{\partial \mathrm{x}}+\frac{\partial \sigma_{\mathrm{yz}}^{(\mathrm{L})}}{\partial \mathrm{y}}\right) \mathrm{dz}+\mathrm{C}_{2}^{(\mathrm{L})}(\mathrm{x}, \mathrm{y}) \\
\sigma_{\mathrm{zz}}^{(\mathrm{L})}=-\int_{\mathrm{z}_{1}}^{\mathrm{z}}\left(\frac{\partial \sigma_{\mathrm{xz}}^{(\mathrm{L})}}{\partial \mathrm{x}}+\frac{\partial \sigma_{\mathrm{yz}}^{(\mathrm{L})}}{\partial \mathrm{y}}\right) \mathrm{dz}+\mathrm{C}_{3}^{(\mathrm{L})}(\mathrm{x}, \mathrm{y})
\end{gathered}
$$

$\tau_{\mathrm{xz}}, \tau_{\mathrm{yz}}$ and $\sigma_{\mathrm{z}}$ are evaluated using the bounding plane criteria and the continuity of stresses.

From the bounding plane criteria we obtain

$$
\tau_{\mathrm{xz}}\left(\mathrm{x}, \mathrm{y},-\frac{\mathrm{h}}{2}\right)=0 ; \tau_{\mathrm{yz}}\left(\mathrm{x}, \mathrm{y},-\frac{\mathrm{h}}{2}\right)=0 ; \sigma_{\mathrm{z}}\left(\mathrm{x}, \mathrm{y}, \frac{-\mathrm{h}}{2}\right)=0
$$

Following conditions are used For Continuity of stress

$$
\begin{aligned}
& \tau_{\mathrm{xz}}^{\mathrm{L}}\left(\mathrm{x}, \mathrm{y}, \mathrm{z}_{\mathrm{L}+1}\right)=\tau_{\mathrm{xz}}^{\mathrm{L}+1}\left(\mathrm{x}, \mathrm{y}, \mathrm{z}_{\mathrm{L}+1}\right) ; \quad \tau_{\mathrm{yz}}^{\mathrm{L}}\left(\mathrm{x}, \mathrm{y}, \mathrm{z}_{\mathrm{L}+1}\right)=\tau_{\mathrm{yz}}^{\mathrm{L}+1}\left(\mathrm{x}, \mathrm{y}, \mathrm{z}_{\mathrm{L}+1}\right) \\
& \sigma_{\mathrm{z}}^{\mathrm{L}}\left(\mathrm{x}, \mathrm{y}, \mathrm{z}_{\mathrm{L}+1}\right)=\sigma_{\mathrm{z}}^{\mathrm{L}+1}\left(\mathrm{x}, \mathrm{y}, \mathrm{z}_{\mathrm{L}+1}\right)
\end{aligned}
$$

\subsection{Transverse Stress $\left(\tau_{\mathrm{xz}}\right)$}

From Equation (23) we can write the value of transverse stress $\tau_{x z}$ for any $\mathrm{L}^{\text {th }}$ layer as

$$
\tau_{x z}^{(L)}=-\int_{Z_{L}}^{z}\left(\frac{\partial \sigma_{x}^{(L)}}{\partial x}+\frac{\partial \tau_{x y}^{(L)}}{\partial y}\right) d z
$$

Substituting the values of $\sigma_{x}$ and $\tau_{x y}$ in terms of strains from equations (2) and (6) in to equation (10) we get

$$
\tau_{x z}^{(L)}=-\int_{Z_{L}}^{Z}\left\{\frac{\partial}{\partial x}\left[Q_{11} \varepsilon_{x}+Q_{12} \varepsilon_{y}+Q_{13} \varepsilon_{z}+Q_{14} \gamma_{x y}\right]+\frac{\partial}{\partial y}\left[Q_{41} \varepsilon_{x}+Q_{42} \varepsilon_{y}+Q_{43} \varepsilon_{z}+Q_{44} \gamma_{x y}\right]\right\} d z
$$

And after solving above equation and using boundary conditions:

At the bounding plane we get

$$
Z=Z_{L}=-\frac{h}{2} \quad \tau_{x z}=0 \quad \text { i.e. } \tau_{x z}\left(x, y,-\frac{h}{2}\right)=0
$$

Condition for continuity of stress at layer interface are applied. The value of $\tau_{x z}$ is given by:

$$
\begin{aligned}
& \tau_{x z}^{(L)}\left(x, y, z_{L}\right)=-\left\{Q_{11} H_{1} \frac{\partial^{2} u_{0}}{\partial x^{2}}+Q_{11} H_{2} \frac{\partial^{2} \theta_{x}}{\partial x^{2}}+Q_{11} H_{3} \frac{\partial^{2} u_{0}^{*}}{\partial x^{2}}+Q_{11} H_{4} \frac{\partial^{2} \theta_{x}^{*}}{\partial x^{2}}+Q_{12} H_{1} \frac{\partial^{2} v_{0}}{\partial x \partial y}\right. \\
& +Q_{12} H_{2} \frac{\partial^{2} \theta_{y}}{\partial x \partial y}+Q_{12} H_{3} \frac{\partial^{2} v_{0}^{*}}{\partial x \partial y}+Q_{12} H_{4} \frac{\partial^{2} \theta_{y}^{*}}{\partial x \partial y}+Q_{13} H_{1} \frac{\partial \theta_{z}}{\partial x}+Q_{13} H_{2} 2 \frac{\partial w_{0}^{*}}{\partial x}+Q_{13} 3 . H_{3} \frac{\partial \theta_{z}^{*}}{\partial x}
\end{aligned}
$$




$$
\begin{aligned}
& +Q_{14} H_{1} \frac{\partial^{2} u_{0}}{\partial x \partial y}+Q_{14} H_{2} \frac{\partial^{2} \theta_{x}}{\partial x \partial y}+Q_{14} H_{3} \frac{\partial^{2} u_{0}^{*}}{\partial x \partial y}+Q_{14} H_{4} \frac{\partial^{2} \theta_{x}^{*}}{\partial x \partial y}+Q_{14} H_{1} \frac{\partial^{2} v_{0}}{\partial x^{2}} \\
& +Q_{14} H_{2} \frac{\partial^{2} \theta_{y}}{\partial x^{2}}+Q_{14} H_{3} \frac{\partial^{2} v_{0}^{*}}{\partial x^{2}}+Q_{14} H_{4} \frac{\partial^{2} \theta_{y}^{*}}{\partial x^{2}}+Q_{41} H_{1} \frac{\partial^{2} u_{0}}{\partial x \partial y}+Q_{41} H_{2} \frac{\partial^{2} \theta_{x}}{\partial x \partial y}+Q_{41} H_{3} \frac{\partial^{2} u_{0}^{*}}{\partial x \partial y} \\
& +Q_{41} H_{4} \frac{\partial^{2} \theta_{x}^{*}}{\partial x \partial y}+Q_{42} H_{1} \frac{\partial^{2} v_{0}}{\partial y^{2}}+Q_{42} H_{2} \frac{\partial^{2} \theta_{y}}{\partial y^{2}}+Q_{42} H_{3} \frac{\partial^{2} v_{0}^{*}}{\partial y^{2}}+Q_{42} H_{4} \frac{\partial^{2} \theta_{y}^{*}}{\partial y^{2}}+Q_{43} H_{1} \frac{\partial \theta_{z}}{\partial y} \\
& +Q_{43} 2 H_{2} \frac{\partial^{2} w_{0}^{*}}{\partial y}+Q_{43} H_{3} 3 \frac{\partial \theta_{z}^{*}}{\partial y}+Q_{44} H_{1} \frac{\partial^{2} u_{0}}{\partial y^{2}}+Q_{44} H_{2} \frac{\partial^{2} \theta_{x}}{\partial y^{2}}+Q_{44} H_{3} \frac{\partial^{2} u_{0}^{*}}{\partial y^{2}} \\
& \left.+Q_{44} H_{4} \frac{\partial^{2} \theta_{x}^{*}}{\partial y^{2}}+Q_{44} H_{1} \frac{\partial^{2} v_{0}}{\partial x \partial y}+Q_{44} H_{2} \frac{\partial^{2} \theta_{y}}{\partial x \partial y}+Q_{44} H_{3} \frac{\partial^{2} v_{0}^{*}}{\partial x \partial y}+Q_{44} H_{4} \frac{\partial^{2} \theta_{y}^{*}}{\partial x \partial y}\right\} \\
& -\left\{Q_{11} H_{1} \frac{\partial^{2} u_{0}}{\partial x^{2}}+Q_{11} H_{2} \frac{\partial^{2} \theta_{x}}{\partial x^{2}}+Q_{11} H_{3} \frac{\partial^{2} u_{0}^{*}}{\partial x^{2}}+Q_{11} H_{4} \frac{\partial^{2} \theta_{x}^{*}}{\partial x^{2}}+Q_{12} H_{1} \frac{\partial^{2} v_{0}}{\partial x \partial y}+Q_{12} H_{2} \frac{\partial^{2} \theta_{y}}{\partial x \partial y}\right. \\
& +Q_{12} H_{3} \frac{\partial^{2} v_{0}^{*}}{\partial x \partial y}+Q_{12} H_{4} \frac{\partial^{2} \theta_{y}^{*}}{\partial x \partial y}+Q_{13} H_{1} \frac{\partial \theta_{z}}{\partial x}+Q_{13} H_{2} 2 \frac{\partial w_{0}^{*}}{\partial x}+Q_{13} 3 . H_{3} \frac{\partial \theta_{z}^{*}}{\partial x}+Q_{14} H_{1} \frac{\partial^{2} u_{0}}{\partial x \partial y} \\
& +Q_{14} H_{2} \frac{\partial^{2} \theta_{x}}{\partial x \partial y}+Q_{14} H_{3} \frac{\partial^{2} u_{0}^{*}}{\partial x \partial y}+Q_{14} H_{4} \frac{\partial^{2} \theta_{x}^{*}}{\partial x \partial y}+Q_{14} H_{1} \frac{\partial^{2} v_{0}}{\partial x^{2}}+Q_{14} H_{2} \frac{\partial^{2} \theta_{y}}{\partial x^{2}}+Q_{14} H_{3} \frac{\partial^{2} v_{0}^{*}}{\partial x^{2}} \\
& +Q_{14} H_{4} \frac{\partial^{2} \theta_{y}^{*}}{\partial x^{2}}+Q_{41} H_{1} \frac{\partial^{2} u_{0}}{\partial x \partial y}+Q_{41} H_{2} \frac{\partial^{2} \theta_{x}}{\partial x \partial y}+Q_{41} H_{3} \frac{\partial^{2} u_{0}^{*}}{\partial x \partial y}+Q_{41} H_{4} \frac{\partial^{2} \theta_{x}^{*}}{\partial x \partial y} \\
& +Q_{42} H_{1} \frac{\partial^{2} v_{0}}{\partial y^{2}}+Q_{42} H_{2} \frac{\partial^{2} \theta_{y}}{\partial y^{2}}+Q_{42} H_{3} \frac{\partial^{2} v_{0}^{*}}{\partial y^{2}}+Q_{42} H_{4} \frac{\partial^{2} \theta_{y}^{*}}{\partial y^{2}}+Q_{43} H_{1} \frac{\partial \theta_{z}}{\partial y}+Q_{43} 2 H_{2} \frac{\partial^{2} w_{0}^{*}}{\partial y} \\
& +Q_{43} H_{3} 3 \frac{\partial \theta_{z}^{*}}{\partial y}+Q_{44} H_{1} \frac{\partial^{2} u_{0}}{\partial y^{2}}+Q_{44} H_{2} \frac{\partial^{2} \theta_{x}}{\partial y^{2}}+Q_{44} H_{3} \frac{\partial^{2} u_{0}^{*}}{\partial y^{2}}+Q_{44} H_{4} \frac{\partial^{2} \theta_{x}^{*}}{\partial y^{2}} \\
& \left.+Q_{44} H_{1} \frac{\partial^{2} v_{0}}{\partial x \partial y}+Q_{44} H_{2} \frac{\partial^{2} \theta_{y}}{\partial x \partial y}+Q_{44} H_{3} \frac{\partial^{2} v_{0}^{*}}{\partial x \partial y}+Q_{44} H_{4} \frac{\partial^{2} \theta_{y}^{*}}{\partial x \partial y}\right\} \\
& \mathrm{H}_{\mathrm{i}}=\frac{1}{\mathrm{i}}\left(\mathrm{z}_{\mathrm{L}+1}^{\mathrm{i}}-\mathrm{z}_{\mathrm{L}}^{\mathrm{i}}\right) \ldots \ldots \ldots \ldots \ldots . . .(\mathrm{i}=1 \text { to } 7)
\end{aligned}
$$

Similarly the final expressions for the computation of $\tau_{y z}, \sigma_{z}$ are given as below

\subsection{Transverse Stress $\left({ }^{\tau_{y z}}\right)$}

The general expression for shear stress $\tau_{y z}^{(L)}$ for any $\mathrm{L}^{\text {th }}$ layer is given by

$$
\begin{aligned}
& \tau_{y z}^{(L)}\left(x, y, z_{L}\right)=-\left\{Q_{41} H_{1} \frac{\partial^{2} u_{0}}{\partial x^{2}}+Q_{41} H_{2} \frac{\partial^{2} \theta_{x}}{\partial x^{2}}+Q_{41} H_{3} \frac{\partial^{2} u_{0}^{*}}{\partial x^{2}}+Q_{41} H_{4} \frac{\partial^{2} \theta_{x}^{*}}{\partial x^{2}}+Q_{42} H_{1} \frac{\partial^{2} v_{0}}{\partial x \partial y}\right. \\
& +Q_{42} H_{2} \frac{\partial^{2} \theta_{y}}{\partial x \partial y}+Q_{42} H_{3} \frac{\partial^{2} v_{0}^{*}}{\partial x \partial y}+Q_{42} H_{4} \frac{\partial^{2} \theta_{y}^{*}}{\partial x \partial y}+Q_{43} H_{1} \frac{\partial \theta_{z}}{\partial x}+Q_{43} H_{2} 2 \frac{\partial w_{0}^{*}}{\partial x}+Q_{43} 3 . H_{3} \frac{\partial \theta_{z}^{*}}{\partial x} \\
& +Q_{44} H_{1} \frac{\partial^{2} u_{0}}{\partial x \partial y}+Q_{44} H_{2} \frac{\partial^{2} \theta_{x}}{\partial x \partial y}+Q_{44} H_{3} \frac{\partial^{2} u_{0}^{*}}{\partial x \partial y}+Q_{44} H_{4} \frac{\partial^{2} \theta_{x}^{*}}{\partial x \partial y}+Q_{44} H_{1} \frac{\partial^{2} v_{0}}{\partial x^{2}}+Q_{44} H_{2} \frac{\partial^{2} \theta_{y}}{\partial x^{2}}
\end{aligned}
$$


Higher Order Refined Computational Model using Post Processing for the Transverse Shear and Transverse Normal Stress in Composite Plates

$$
\begin{aligned}
& +Q_{44} H_{3} \frac{\partial^{2} v_{0}^{*}}{\partial x^{2}}+Q_{44} H_{4} \frac{\partial^{2} \theta_{y}^{*}}{\partial x^{2}}+Q_{21} H_{1} \frac{\partial^{2} u_{0}}{\partial x \partial y}+Q_{21} H_{2} \frac{\partial^{2} \theta_{x}}{\partial x \partial y}+Q_{21} H_{3} \frac{\partial^{2} u_{0}^{*}}{\partial x \partial y}+Q_{21} H_{4} \frac{\partial^{2} \theta_{x}^{*}}{\partial x \partial y} \\
& +Q_{22} H_{1} \frac{\partial^{2} v_{0}}{\partial y^{2}}+Q_{22} H_{2} \frac{\partial^{2} \theta_{y}}{\partial y^{2}}+Q_{22} H_{3} \frac{\partial^{2} v_{0}^{*}}{\partial y^{2}}+Q_{22} H_{4} \frac{\partial^{2} \theta_{y}^{*}}{\partial y^{2}}+Q_{23} H_{1} \frac{\partial \theta_{z}}{\partial y} \\
& +Q_{23} 2 H_{2} \frac{\partial^{2} w_{0}^{*}}{\partial y}+Q_{23} H_{3} 3 \frac{\partial \theta_{z}^{*}}{\partial y}+Q_{24} H_{1} \frac{\partial^{2} u_{0}}{\partial y^{2}}+Q_{24} H_{2} \frac{\partial^{2} \theta_{x}}{\partial y^{2}}+Q_{24} H_{3} \frac{\partial^{2} u_{0}^{*}}{\partial y^{2}} \\
& \left.+Q_{24} H_{4} \frac{\partial^{2} \theta_{x}^{*}}{\partial y^{2}}+Q_{24} H_{1} \frac{\partial^{2} v_{0}}{\partial x \partial y}+Q_{24} H_{2} \frac{\partial^{2} \theta_{y}}{\partial x \partial y}+Q_{24} H_{3} \frac{\partial^{2} v_{0}^{*}}{\partial x \partial y}+Q_{44} H_{4} \frac{\partial^{2} \theta_{y}^{*}}{\partial x \partial y}\right\} \\
& -\left\{Q_{41} H_{1} \frac{\partial^{2} u_{0}}{\partial x^{2}}+Q_{41} H_{2} \frac{\partial^{2} \theta_{x}}{\partial x^{2}}+Q_{41} H_{3} \frac{\partial^{2} u_{0}^{*}}{\partial x^{2}}+Q_{41} H_{4} \frac{\partial^{2} \theta_{x}^{*}}{\partial x^{2}}+Q_{42} H_{1} \frac{\partial^{2} v_{0}}{\partial x \partial y}+Q_{42} H_{2} \frac{\partial^{2} \theta_{y}}{\partial x \partial y}\right. \\
& +Q_{42} H_{3} \frac{\partial^{2} v_{0}^{*}}{\partial x \partial y}+Q_{42} H_{4} \frac{\partial^{2} \theta_{y}^{*}}{\partial x \partial y}+Q_{43} H_{1} \frac{\partial \theta_{z}}{\partial x}+Q_{43} H_{2} 2 \frac{\partial w_{0}^{*}}{\partial x}+Q_{43} 3 . H_{3} \frac{\partial \theta_{z}^{*}}{\partial x}+Q_{44} H_{1} \frac{\partial^{2} u_{0}}{\partial x \partial y} \\
& +Q_{44} H_{2} \frac{\partial^{2} \theta_{x}}{\partial x \partial y}+Q_{44} H_{3} \frac{\partial^{2} u_{0}^{*}}{\partial x \partial y}+Q_{44} H_{4} \frac{\partial^{2} \theta_{x}^{*}}{\partial x \partial y}+Q_{44} H_{1} \frac{\partial^{2} v_{0}}{\partial x^{2}}+Q_{44} H_{2} \frac{\partial^{2} \theta_{y}}{\partial x^{2}}+Q_{44} H_{3} \frac{\partial^{2} v_{0}^{*}}{\partial x^{2}} \\
& +Q_{44} H_{4} \frac{\partial^{2} \theta_{y}^{*}}{\partial x^{2}}+Q_{21} H_{1} \frac{\partial^{2} u_{0}}{\partial x \partial y}+Q_{21} H_{2} \frac{\partial^{2} \theta_{x}}{\partial x \partial y}+Q_{21} H_{3} \frac{\partial^{2} u_{0}^{*}}{\partial x \partial y}+Q_{21} H_{4} \frac{\partial^{2} \theta_{x}^{*}}{\partial x \partial y}+Q_{22} H_{1} \frac{\partial^{2} v_{0}}{\partial y^{2}} \\
& +Q_{22} H_{2} \frac{\partial^{2} \theta_{y}}{\partial y^{2}}+Q_{22} H_{3} \frac{\partial^{2} v_{0}^{*}}{\partial y^{2}}+Q_{22} H_{4} \frac{\partial^{2} \theta_{y}^{*}}{\partial y^{2}}+Q_{23} H_{1} \frac{\partial \theta_{z}}{\partial y}+Q_{23} 2 H_{2} \frac{\partial^{2} w_{0}^{*}}{\partial y}+Q_{23} H_{3} 3 \frac{\partial \theta_{z}^{*}}{\partial y} \\
& +Q_{24} H_{1} \frac{\partial^{2} u_{0}}{\partial y^{2}}+Q_{24} H_{2} \frac{\partial^{2} \theta_{x}}{\partial y^{2}}+Q_{24} H_{3} \frac{\partial^{2} u_{0}^{*}}{\partial y^{2}}+Q_{24} H_{4} \frac{\partial^{2} \theta_{x}^{*}}{\partial y^{2}}+Q_{24} H_{1} \frac{\partial^{2} v_{0}}{\partial x \partial y} \\
& \left.+Q_{24} H_{2} \frac{\partial^{2} \theta_{y}}{\partial x \partial y}+Q_{24} H_{3} \frac{\partial^{2} v_{0}^{*}}{\partial x \partial y}+Q_{24} H_{4} \frac{\partial^{2} \theta_{y}^{*}}{\partial x \partial y}\right\}
\end{aligned}
$$

\subsection{Transverse Stress $\left(\sigma_{\mathrm{z}}\right)$}

The general expression for transverse stress $\sigma_{\mathrm{z}}$ for $\mathrm{L}^{\text {th }}$ layer becomes

$$
\begin{aligned}
& \sigma_{z}(x, y, z)=-\left\{Q _ { 6 5 } \left\{H_{1}\left(\frac{\partial \theta_{y}}{\partial x}+\frac{\partial^{2} w_{0}}{\partial x \partial y}\right)+H_{2}\left(2 \frac{\partial v_{0}^{*}}{\partial x}+\frac{\partial^{2} \theta_{z}}{\partial x \partial y}\right)\right.\right. \\
& \left.+H_{3}\left(3 \frac{\partial \theta_{y}^{*}}{\partial x}+\frac{\partial^{2} w_{0}^{*}}{\partial x \partial y}\right)+H_{4}\left(\frac{\partial^{2} \theta_{z}^{*}}{\partial x \partial y}\right)\right\} \\
& +Q_{66}\left\{H_{1}\left(\frac{\partial \theta_{x}}{\partial x}+\frac{\partial^{2} w_{0}}{\partial x^{2}}\right)+H_{2}\left(2 \frac{\partial u_{0}^{*}}{\partial x}+\frac{\partial^{2} \theta_{z}}{\partial x^{2}}\right)\right. \\
& \left.+H_{3}\left(3 \frac{\partial \theta_{x}^{*}}{\partial x}+\frac{\partial^{2} w_{0}^{*}}{\partial x^{2}}\right)+H_{4}\left(\frac{\partial^{2} \theta_{z}^{*}}{\partial x^{2}}\right)\right\} \\
& +Q_{55}\left\{H_{1}\left(\frac{\partial \theta_{y}}{\partial y}+\frac{\partial^{2} w_{0}}{\partial y^{2}}\right)+H_{2}\left(2 \frac{\partial v_{0}^{*}}{\partial y}+\frac{\partial^{2} \theta_{z}}{\partial y^{2}}\right)\right. \\
& \left.+H_{3}\left(3 \frac{\partial \theta_{y}^{*}}{\partial y}+\frac{\partial^{2} w_{0}^{*}}{\partial y^{2}}\right)+H_{4}\left(\frac{\partial^{2} \theta_{z}^{*}}{\partial y^{2}}\right)\right\}
\end{aligned}
$$




$$
\begin{aligned}
& +Q_{56}\left\{H_{1}\left(\frac{\partial \theta_{x}}{\partial y}+\frac{\partial^{2} w_{0}}{\partial x \partial y}\right)+H_{2}\left(2 \frac{\partial u_{0}^{*}}{\partial y}+\frac{\partial^{2} \theta_{z}}{\partial x \partial y}\right)\right. \\
& \left.\left.+H_{3}\left(3 \frac{\partial \theta_{x}^{*}}{\partial y}+\frac{\partial^{2} w_{0}^{*}}{\partial x \partial y}\right)+H_{4}\left(\frac{\partial^{2} \theta_{z}^{*}}{\partial x \partial y}\right)\right\}\right\} \\
& -\left\{Q _ { 6 5 } \left\{H_{1}\left(\frac{\partial \theta_{y}}{\partial x}+\frac{\partial^{2} w_{0}}{\partial x \partial y}\right)+H_{2}\left(2 \frac{\partial v_{0}^{*}}{\partial x}+\frac{\partial^{2} \theta_{z}}{\partial x \partial y}\right)\right.\right. \\
& \left.+H_{3}\left(3 \frac{\partial \theta_{y}^{*}}{\partial x}+\frac{\partial^{2} w_{0}^{*}}{\partial x \partial y}\right)+H_{4}\left(\frac{\partial^{2} \theta_{z}^{*}}{\partial x \partial y}\right)\right\} \\
& +Q_{66}\left\{H_{1}\left(\frac{\partial \theta_{x}}{\partial x}+\frac{\partial^{2} w_{0}}{\partial x^{2}}\right)+H_{2}\left(2 \frac{\partial u_{0}^{*}}{\partial x}+\frac{\partial^{2} \theta_{z}}{\partial x^{2}}\right)\right. \\
& \left.+H_{3}\left(3 \frac{\partial \theta_{x}^{*}}{\partial x}+\frac{\partial^{2} w_{0}^{*}}{\partial x^{2}}\right)+H_{4}\left(\frac{\partial^{2} \theta_{z}^{*}}{\partial x^{2}}\right)\right\} \\
& +Q_{55}\left\{H_{1}\left(\frac{\partial \theta_{y}}{\partial y}+\frac{\partial^{2} w_{0}}{\partial y^{2}}\right)+H_{2}\left(2 \frac{\partial v_{0}^{*}}{\partial y}+\frac{\partial^{2} \theta_{z}}{\partial y^{2}}\right)\right. \\
& \left.+H_{3}\left(3 \frac{\partial \theta_{y}^{*}}{\partial y}+\frac{\partial^{2} w_{0}^{*}}{\partial y^{2}}\right)+H_{4}\left(\frac{\partial^{2} \theta_{z}^{*}}{\partial y^{2}}\right)\right\} \\
& +Q_{56}\left\{H_{1}\left(\frac{\partial \theta_{x}}{\partial y}+\frac{\partial^{2} w_{0}}{\partial x \partial y}\right)+H_{2}\left(2 \frac{\partial u_{0}^{*}}{\partial y}+\frac{\partial^{2} \theta_{z}}{\partial x \partial y}\right)\right. \\
& \left.\left.+H_{3}\left(3 \frac{\partial \theta_{x}^{*}}{\partial y}+\frac{\partial^{2} w_{0}^{*}}{\partial x \partial y}\right)+H_{4}\left(\frac{\partial^{2} \theta_{z}^{*}}{\partial x \partial y}\right)\right\}\right\}
\end{aligned}
$$

\section{NUMERICAL RESULTS AND DISCUSSIONS}

Two numerical examples are solved and presented.

\subsection{Material Properties}

The following sets of material constants are used.

\section{Material 1}

$\mathrm{E}_{1}=25 \times 10^{6} \mathrm{ps}(276 \mathrm{GPa}) \quad \mathrm{E}_{2}=\mathrm{E}_{3}=1 \times 10^{6} \mathrm{ps}(6.895 \mathrm{Gpa}) \mathrm{G}_{12}=\mathrm{G}_{13}=0.5 \times 10^{6} \mathrm{ps}(3.45$ GPa ) $G_{23}=0.2 \times 10^{6} \mathrm{ps}(4.12 \mathrm{GPa})$

$$
v_{12}=v_{23}=v_{13}=0.25
$$

Following non-dimensional forms are used to present the results.

$$
\begin{aligned}
& \bar{\sigma}_{\mathrm{x}}=\sigma_{\mathrm{x}}\left(\frac{\mathrm{h}^{2}}{\mathrm{P}_{\mathrm{o}} \mathrm{a}^{2}}\right) \bar{\sigma}_{\mathrm{y}}=\sigma_{\mathrm{y}}\left(\frac{\mathrm{h}^{2}}{\mathrm{P}_{\mathrm{o}} \mathrm{a}^{2}}\right) \bar{\tau}_{\mathrm{xy}}=\tau_{\mathrm{xy}}\left(\frac{\mathrm{h}^{2}}{\mathrm{P}_{\mathrm{o}} \mathrm{a}^{2}}\right) \\
& \bar{\tau}_{\mathrm{xz}}=\tau_{\mathrm{xz}}\left(\frac{\mathrm{h}}{\mathrm{P}_{0} \mathrm{a}}\right) \bar{\tau}_{\mathrm{yz}}=\tau_{\mathrm{yz}}\left(\frac{\mathrm{h}}{\mathrm{P}_{0} \mathrm{a}}\right)_{\bar{\sigma}_{\mathrm{z}}=\sigma_{\mathrm{z}}\left(\frac{\mathrm{h}^{2}}{\mathrm{P}_{\mathrm{o}} \mathrm{a}^{2}}\right)}
\end{aligned}
$$




\subsection{Numerical Examples}

Example 1: A simply supported, square composite plate is considered. Plate has two layers. Plate is made up of antisymmetric angle ply $(\theta /-\theta)$. Plate is subjected to sinusoidal transverse load. The layers are of equal thickness. Material Set 1 is used. The nondimensionalized maximum in plane stresses $\left(\bar{\sigma}_{x}, \bar{\sigma}_{y}, \bar{\tau}_{x y}\right)$ and transverse stresses $\left(\bar{\tau}_{x z}, \bar{\tau}_{y z}\right)$ for plates with varying $\mathrm{a} / \mathrm{h}$ ratios and fibre orientations are given in Table 1 to Table 3 . The solutions using 3D elasticity theory [14] and other shear deformation theories [15,16] already reported in literature are used for comparison. In thick plates, it has been found that for all the fibre orientations considered the inplane and transverse stress values obtained using the present model are very close to $3 \mathrm{D}$ elasticity values. For plate with $\mathrm{a} / \mathrm{h}$ ratio equal to 4 and fibre orientation equal to $30^{\circ}$ the values of $\bar{\tau}_{x z}(a / 2,0)$ predicted by the present model, RSDT and HSDT -5 are respectively $10.34 \%, 19.08 \%$ and $35.73 \%$ higher as compared to 3D elasticity solutions. Similarly for the same plate the values of $\bar{\tau}_{y z}(0, a / 2)$ predicted by the present and other models are respectively $8.06 \%, 14.98 \%$ and $28.34 \%$ higher as compared to the solutions reported using 3D elasticity theory. Similar patterns in the percentage difference between the models can be observed in all other stress values also for all the fibre orientations considered. Figures 2 show the through the thickness variation of nondimensionalized transverse stress $\bar{\tau}_{x z}, \bar{\tau}_{y z}$ at $(\mathrm{a} / 2,0)$ for plates with a/h ratios 4 and fibre orientation equal to $15^{\circ}$. Figures 3 show the through the thickness variation of nondimensionalized transverse stress at $(\mathrm{a} / 2,0)$ for plates with a/h ratios 10 and fibre orientation equal to $15^{\circ}$. The plot of through the thickness variation of transverse normal stress $\left(\bar{\sigma}_{z}\right)$ for plates with a/h ratios 4 and 10 is given in Figure 4. Table 5 gives comparison of transverse stresses with the present model for 4 layer and 8 layer composite plate.
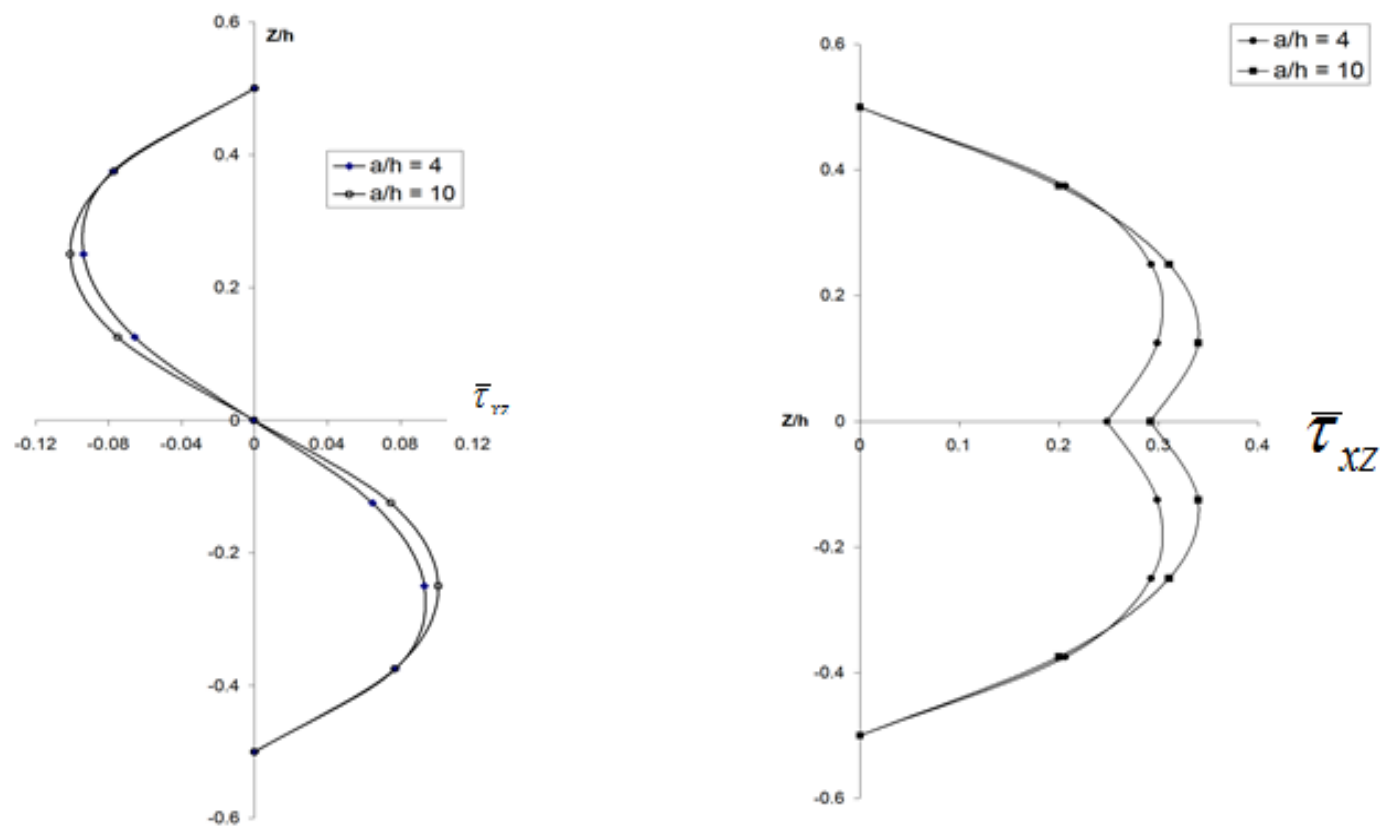

Figure 2 Variation of transverse shear $\bar{\tau}_{x z}$ stress $\left(\bar{\tau}_{x z}\right) \quad$ Figure 3 Variation of transverse shear stress $\left(\bar{\tau}_{x z}\right)$

Example 2: A simply supported, rectangular plate $(b=3 a)$ composite plate is used. Type of plate, material of plate, Number of layers and their thickness along with loading on the plate is same as given in Example 1. The variation of nondimensionalized maximum stresses for plates with varying a/h ratios is considered. a/h ratios 4 and 10 with fibre angles $\theta=15^{\circ}, 30^{\circ}$ 
and $45^{\circ}$ are tabulated in Table 4. Comparison of results obtained using the present model with the 3D elasticity and other models solutions already available $[14,15,16]$ clearly indicates that for all the parameters considered in the present investigation, the present model predicts all the stresses very much closer to 3D elasticity solutions. For a particular value of fibre orientation $\theta=30^{\circ}$ and a/h value equal to 4 , the value of $\bar{\tau}_{x z}(a / 2,0)$ obtained using the present model, RSDT [15] and HSDT-5 [16] are respectively 14.0\%, 25.82\% and 56.89\% higher and the values of $\bar{\tau}_{y z}(0, b / 2)$ are respectively $4.5 \%, 6.0 \%$ and $9.98 \%$ higher than the $3 \mathrm{D}$ elasticity solutions.

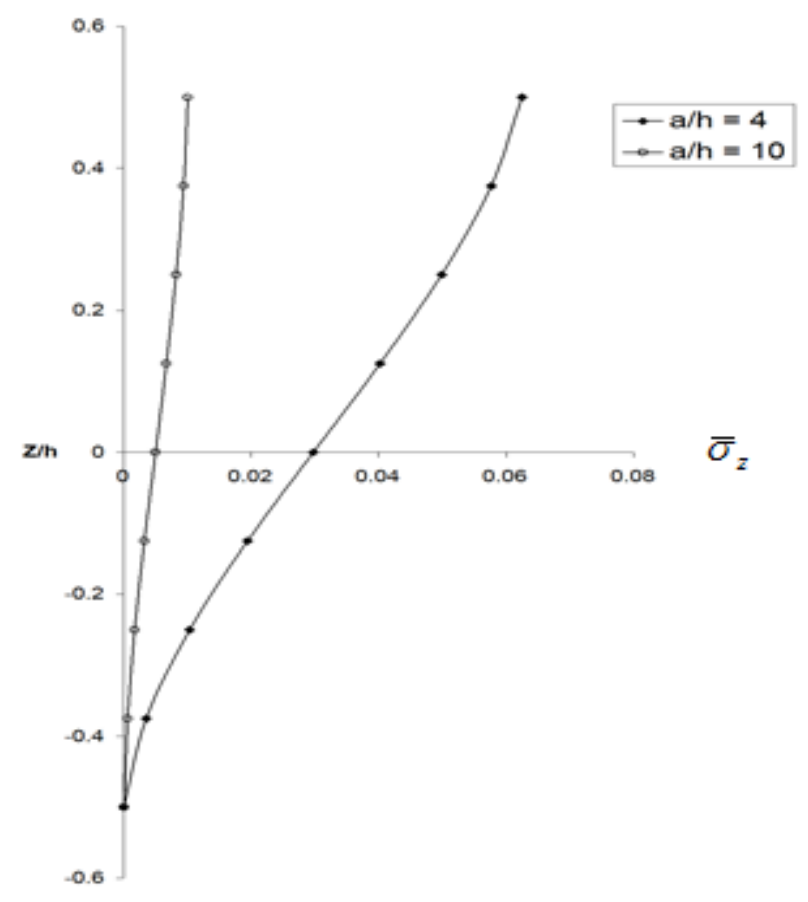

Figure 4 Variation of transverse normal stress $\left(\bar{\sigma}_{z}\right)$

Table 1 Non-dimensionalised maximum stresses in simply supported, two layered (15/-15) angle ply, square laminate

\begin{tabular}{|c|c|c|c|c|c|c|c|c|}
\hline $\mathrm{a} / \mathrm{h}$ & Theory & $\bar{\sigma}_{x}(\mathrm{a} / 2, \mathrm{a} / 2)$ & $\bar{\sigma}_{y}(\mathrm{a} / 2, \mathrm{a} / 2)$ & $\bar{\tau}_{x y}(\mathrm{a} / 2, \mathrm{a} / 2)$ & $\bar{\tau}_{\mathrm{xz}}(\mathrm{a} / 2,2)$ & $\bar{\tau}_{y z}(a / 2,0)$ & $\bar{\tau}_{\mathrm{xz}}(0, \mathrm{a} / 2)$ & $\bar{\tau}_{\mathrm{yz}}(0, \mathrm{a} / 2)$ \\
\hline \multirow{4}{*}{4} & Present & 0.6559 & 0.1201 & 0.1451 & 0.0907 & 0.0834 & 0.2991 & 0.0403 \\
\hline & 3 D-Elasticity [16] & 0.6645 & 0.1135 & 0.1489 & 0.0841 & 0.0960 & 0.3145 & 0.0399 \\
\hline & RSDT* [17] & 0.5884 & 0.0980 & 0.1305 & 0.1034 & 0.0929 & 0.3186 & 0.0428 \\
\hline & HSDT-5 ${ }^{\#}[17]$ & 0.7032 & 0.1064 & 0.1633 & 0.1282 & 0.0899 & 0.3061 & 0.0465 \\
\hline \multirow{4}{*}{10} & Present & 0.5606 & 0.0810 & 0.1243 & 0.1001 & 0.0804 & 0.3419 & 0.0412 \\
\hline & 3 D-Elasticity [16] & 0.5633 & 0.0806 & 0.1244 & 0.0991 & 0.0814 & 0.3421 & 0.0412 \\
\hline & $\operatorname{RSDT}^{*}[17]$ & 0.5514 & 0.0778 & 0.1216 & 0.1039 & 0.0807 & 0.3427 & 0.0418 \\
\hline & HSDT-5 $^{\#}[17]$ & 0.5680 & 0.0792 & 0.1264 & 0.1079 & 0.0800 & 0.3133 & 0.0424 \\
\hline \multirow{3}{*}{20} & Present & 0.5463 & 0.0748 & 0.1208 & 0.1015 & 0.0767 & 0.3477 & 0.0411 \\
\hline & $\operatorname{RSDT}^{*}[17]$ & 0.5456 & 0.0741 & 0.1201 & 0.1033 & 0.0780 & 0.3478 & 0.0417 \\
\hline & HSDT-5 ${ }^{\#}[17]$ & 0.5497 & 0.0744 & 0.1213 & 0.1043 & 0.0725 & 0.3152 & 0.0418 \\
\hline \multirow{3}{*}{100} & Present & 0.5415 & 0.0724 & 0.1196 & 0.1019 & 0.0762 & 0.3500 & 0.0412 \\
\hline & $\operatorname{RSDT}^{*}[17]$ & 0.5437 & 0.0728 & 0.1196 & 0.1030 & 0.0771 & 0.3496 & 0.0417 \\
\hline & HSDT-5 $^{\#}[17]$ & 0.5439 & 0.0728 & 0.1196 & 0.1030 & 0.0714 & 0.3159 & 0.0417 \\
\hline
\end{tabular}

*Refined Shear Deformation Theory (RSDT)

\#Higher Order Shear Deformation Theory with 5 degrees of freedom (HSDT-5) 
Higher Order Refined Computational Model using Post Processing for the Transverse Shear and Transverse Normal Stress in Composite Plates

Table 2 Non-dimensionalised maximum stresses in simply supported, two layered (30/-30) square laminate

\begin{tabular}{|c|c|c|c|c|c|c|c|c|}
\hline$a / h$ & Theory & $\bar{\sigma}_{\mathrm{x}}(\mathrm{a} / 2, \mathrm{a} / 2)$ & $\bar{\sigma}_{y}(\mathrm{a} / 2, \mathrm{a} / 2)$ & $\bar{\tau}_{x y}(a / 2, a / 2)$ & $\bar{\tau}_{\mathrm{xz}}(\mathrm{a} / 2,0)$ & $\bar{\tau}_{\mathrm{yz}}(\mathrm{a} / 2,0)$ & $\bar{\tau}_{x z}(0, a / 2)$ & $\bar{\tau}_{\mathrm{yz}}(0, \mathrm{a} / 2)$ \\
\hline \multirow{4}{*}{4} & Present & 0.4747 & 0.2067 & 0.2181 & 0.1504 & 0.1481 & 0.2509 & 0.0938 \\
\hline & 3 D-Elasticity [16] & 0.4945 & 0.2089 & 0.2335 & 0.1363 & 0.1546 & 0.2561 & 0.0868 \\
\hline & $\mathrm{RSDT}^{*}[17]$ & 0.4239 & 0.1763 & 0.1999 & 0.1623 & 0.1584 & 0.2673 & 0.0998 \\
\hline & HSDT-5 $5^{\#}[17]$ & 0.5321 & 0.2104 & 0.2684 & 0.1850 & 0.1581 & 0.2666 & 0.1114 \\
\hline \multirow{4}{*}{10} & Present & 0.4154 & 0.1682 & 0.1989 & 0.1666 & 0.1526 & 0.2634 & 0.1015 \\
\hline & 3 D-Elasticity [16] & 0.4204 & 0.1696 & 0.2018 & 0.1639 & 0.1570 & 0.2699 & 0.1002 \\
\hline & $\mathrm{RSDT}^{*}[17]$ & 0.4090 & 0.1639 & 0.1968 & 0.1703 & 0.1578 & 0.2723 & 0.1035 \\
\hline & HSDT-5 $5^{\#}[17]$ & 0.4267 & 0.1695 & 0.2081 & 0.1741 & 0.1547 & 0.2664 & 0.1055 \\
\hline \multirow{3}{*}{20} & Present & 0.4074 & 0.1626 & 0.1966 & 0.1694 & 0.1532 & 0.2654 & 0.1029 \\
\hline & RSDT* [17] & 0.4070 & 0.1620 & 0.1964 & 0.1715 & 0.1576 & 0.2732 & 0.1041 \\
\hline & HSDT-5 $5^{\#}[17]$ & 0.4114 & 0.1634 & 0.1993 & 0.1725 & 0.1541 & 0.2663 & 0.1046 \\
\hline \multirow{3}{*}{100} & Present & 0.4049 & 0.1608 & 0.1959 & 0.1703 & 0.1534 & 0.2661 & 0.1034 \\
\hline & $\operatorname{RSDT}^{*}[17]$ & 0.4063 & 0.1614 & 0.1963 & 0.1719 & 0.1576 & 0.2735 & 0.1043 \\
\hline & HSDT-5 ${ }^{\#}[17]$ & 0.4065 & 0.1615 & 0.1964 & 0.1720 & 0.1540 & 0.2663 & 0.1043 \\
\hline
\end{tabular}

*Refined Shear Deformation Theory (RSDT)

\#Higher Order Shear Deformation Theory with 5 degrees of freedom (HSDT-5)

Table 3 Non-dimensionalised maximum stresses in simply supported, two layered (45/-45) angle ply, square laminate

\begin{tabular}{|c|c|c|c|c|c|c|c|c|}
\hline $\mathrm{a} / \mathrm{h}$ & Theory & $\bar{\sigma}_{\mathrm{x}}(\mathrm{a} / 2, \mathrm{a} / 2)$ & $\bar{\sigma}_{y}(\mathrm{a} / 2, \mathrm{a} / 2)$ & $\bar{\tau}_{x y}(\mathrm{a} / 2, \mathrm{a} / \mathbf{2})$ & $\bar{\tau}_{\mathrm{xz}}(\mathrm{a} / 2,0)$ & $\bar{\tau}_{\mathrm{yz}}(\mathrm{a} / 2,0)$ & $\bar{\tau}_{x z}(0, a / 2)$ & $\bar{\tau}_{\mathrm{yz}}(0, \mathrm{a} / 2)$ \\
\hline \multirow{4}{*}{4} & Present & 0.2976 & 0.2976 & 0.2144 & 0.1357 & 0.2029 & 0.2029 & 0.1357 \\
\hline & $\begin{array}{c}3 \text { D-Elasticity } \\
{[16]}\end{array}$ & 0.3092 & 0.3092 & 0.2327 & 0.1239 & 0.2062 & 0.2062 & 0.1239 \\
\hline & $\mathrm{RSDT}^{*}[17]$ & 0.2588 & 0.2588 & 0.1956 & 0.1452 & 0.2155 & 0.2155 & 0.1452 \\
\hline & HSDT-5" ${ }^{\#}[17]$ & 0.3392 & 0.3392 & 0.2839 & 0.1618 & 0.2160 & 0.2160 & 0.1618 \\
\hline \multirow{4}{*}{10} & Present & 0.2562 & 0.2562 & 0.1994 & 0.1483 & 0.2119 & 0.2119 & 0.1483 \\
\hline & $\begin{array}{c}3 \text { D-Elasticity } \\
{[16]}\end{array}$ & 0.2594 & 0.2594 & 0.2029 & 0.1461 & 0.2154 & 0.2154 & 0.1461 \\
\hline & RSDT* [17] & 0.2512 & 0.2512 & 0.1975 & 0.1513 & 0.2175 & 0.2175 & 0.1513 \\
\hline & 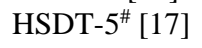 & 0.2646 & 0.2646 & 0.2123 & 0.1542 & 0.2145 & 0.2145 & 0.1542 \\
\hline \multirow{3}{*}{20} & Present & 0.2505 & 0.2505 & 0.1978 & 0.1506 & 0.2133 & 0.2133 & 0.1506 \\
\hline & $\mathrm{RSDT}^{*}[17]$ & 0.2501 & 0.2498 & 0.1981 & 0.1523 & 0.2178 & 0.2178 & 0.1523 \\
\hline & HSDT-5 $5^{\#}[17]$ & 0.2535 & 0.2535 & 0.2016 & 0.1530 & 0.2144 & 0.2144 & 0.1530 \\
\hline \multirow{3}{*}{100} & Present & 0.2487 & 0.2487 & 0.1974 & 0.1513 & 0.2138 & 0.2138 & 0.1513 \\
\hline & RSDT* $^{*}[17]$ & 0.2498 & 0.2498 & 0.1981 & 0.1526 & 0.2179 & 0.2179 & 0.1526 \\
\hline & HSDT $5^{\#}$ [17] & 0.2499 & 0.2499 & 0.1982 & 0.1527 & 0.2143 & 0.2143 & 0.1527 \\
\hline
\end{tabular}

*Refined Shear Deformation Theory (RSDT)

\#Higher Order Shear Deformation Theory with 5 degrees of freedom (HSDT-5) 
Table 4 Non-dimensionalised maximum stresses in simply supported, two layered $(\theta /-\theta)$ angle ply, Rectangular laminate

\begin{tabular}{|c|c|c|c|c|c|c|c|c|c|}
\hline$\theta$ & $\mathrm{a} / \mathrm{h}$ & Theory & $\bar{\sigma}_{\mathrm{x}}(\mathrm{a} / 2, \mathrm{a} / 2)$ & $\overline{\boldsymbol{\sigma}}_{\mathrm{y}}(\mathrm{a} / 2, \mathrm{a} / \mathbf{2})$ & $\bar{\tau}_{x y}(\mathrm{a} / 2, \mathrm{a} / 2)$ & $\bar{\tau}_{x z}(\mathrm{a} / 2,0)$ & $\bar{\tau}_{\mathrm{yz}}(\mathrm{a} / 2,0)$ & $\bar{\tau}_{x z}(0, a / 2)$ & $\bar{\tau}_{y z}(0, a / 2)$ \\
\hline \multirow{7}{*}{$15^{0}$} & \multirow[t]{4}{*}{4} & Present & 0.9562 & 0.0969 & 0.1980 & 0.0511 & 0.0337 & 0.3920 & 0.0609 \\
\hline & & $\begin{array}{c}3 \text { D-Elasticity } \\
{[16]}\end{array}$ & 0.9581 & 0.0911 & 0.2018 & 0.0457 & 0.0365 & 0.3988 & 0.0591 \\
\hline & & RSDT* [17] & 0.8678 & 0.0762 & 0.1805 & 0.0575 & 0.0366 & 0.4021 & 0.0627 \\
\hline & & HSDT $^{-5^{\#}}$ [17] & 1.0111 & 0.0873 & 0.2202 & 0.0717 & 0.0375 & 0.3922 & 0.0650 \\
\hline & \multirow{3}{*}{10} & Present & 0.8085 & 0.0713 & 0.1641 & 0.0570 & 0.0328 & 0.4005 & 0.0598 \\
\hline & & $\mathrm{RSDT}^{*}[17]$ & 0.7973 & 0.0676 & 0.1615 & 0.0583 & 0.0338 & 0.4091 & 0.0602 \\
\hline & & $\mathrm{HSDT}^{-5^{\#}}[17]$ & 0.8186 & 0.0693 & 0.1674 & 0.0607 & 0.0329 & 0.3893 & 0.0605 \\
\hline \multirow{7}{*}{$30^{0}$} & \multirow{4}{*}{4} & Present & 0.9067 & 0.2994 & 0.4113 & 0.1141 & 0.0967 & 0.3806 & 0.1496 \\
\hline & & $\begin{array}{c}3 \text { D-Elasticity } \\
{[16]}\end{array}$ & 0.9282 & 0.3000 & 0.4260 & 0.1029 & 0.0960 & 0.3885 & 0.1427 \\
\hline & & $\mathrm{RSDT}^{*}[17]$ & 0.8514 & 0.2689 & 0.3906 & 0.1225 & 0.1022 & 0.3956 & 0.1532 \\
\hline & & HSDT $-5^{\#}[17]$ & 0.9720 & 0.3087 & 0.4642 & 0.1389 & 0.1060 & 0.3905 & 0.1592 \\
\hline & \multirow{3}{*}{10} & Present & 0.8191 & 0.2614 & 0.3761 & 0.1336 & 0.1069 & 0.3858 & 0.1519 \\
\hline & & $\mathrm{RSDT}^{*}$ [17] & 0.8120 & 0.2569 & 0.3737 & 0.1358 & 0.1095 & 0.3958 & 0.1530 \\
\hline & & HSDT $^{-5^{\#}}{ }^{[17]}$ & 0.8310 & 0.2632 & 0.3853 & 0.1388 & 0.1091 & 0.3877 & 0.1540 \\
\hline \multirow{7}{*}{$45^{0}$} & \multirow{5}{*}{4} & Present & 0.7314 & 0.5862 & 0.5020 & 0.1461 & 0.1976 & 0.3445 & 0.2097 \\
\hline & & $\begin{array}{c}3 \text { D-Elasticity } \\
{[16]}\end{array}$ & 0.7427 & 0.5943 & 0.5182 & 0.1369 & 0.1956 & 0.3564 & 0.2009 \\
\hline & & RSDT* $^{*}[17]$ & 0.6949 & 0.5512 & 0.4861 & 0.1531 & 0.2050 & 0.3609 & 0.2160 \\
\hline & & HSDT-5" [17] & 0.7731 & 0.6297 & 0.5702 & 0.1646 & 0.2076 & 0.3541 & 0.2259 \\
\hline & & Present & 0.6624 & 0.5352 & 0.4695 & 0.1650 & 0.2200 & 0.3465 & 0.2164 \\
\hline & \multirow[t]{2}{*}{10} & $\mathrm{RSDT}^{*}[17]$ & 0.6586 & 0.5313 & 0.4687 & 0.1668 & 0.2230 & 0.3573 & 0.2182 \\
\hline & & HSDT-5" [17] & 0.6719 & 0.5441 & 0.4824 & 0.1688 & 0.2211 & 0.3487 & 0.2199 \\
\hline
\end{tabular}

Table 5 Non-dimensionalised maximum stresses in a four layered $(\theta /-\theta)_{2}$ and six $(\theta /-\theta)_{3}$ layered square laminate plate

\begin{tabular}{|c|c|c|c|c|c|c|c|}
\hline$\theta$ & Theory & $\bar{w}$ & $\bar{\sigma}_{x}(a / 2, a / 2)$ & $\bar{\sigma}_{y}(\mathrm{a} / \mathbf{2}, \mathrm{a} / \mathbf{2})$ & $\bar{\tau}_{x y}(0,0)$ & $\overline{\boldsymbol{\tau}}_{\mathrm{xz}}(\mathbf{0 , a / 2})$ & $\bar{\tau}_{\mathrm{yz}}(\mathrm{a} / 2,0)$ \\
\hline & \multicolumn{7}{|c|}{ Four Layered } \\
\hline \multirow{2}{*}{15} & Present & 0.5957 & 0.4396 & 0.0621 & 0.0658 & 0.4124 & 0.1069 \\
\hline & 3 D-Elasticity [16] & 0.6150 & 0.4453 & 0.0615 & 0.0674 & 0.4116 & 0.1074 \\
\hline \multirow{2}{*}{30} & Present & 0.5136 & 0.2710 & 0.1078 & 0.1262 & 0.3447 & 0.2027 \\
\hline & 3 D-Elasticity [16] & 0.5619 & 0.2833 & 0.1115 & 0.1331 & 0.3381 & 0.1995 \\
\hline \multirow[t]{2}{*}{45} & Present & 0.4826 & 0.1655 & 0.1655 & 0.1533 & 0.2756 & 0.2756 \\
\hline & 3 D-Elasticity [16] & 0.5430 & 0.1749 & 0.1749 & 0.1642 & 0.2684 & 0.2684 \\
\hline \multirow{3}{*}{15} & \multicolumn{7}{|c|}{ Six Layered } \\
\hline & Present & 0.5649 & 0.4277 & 0.0595 & 0.0680 & 0.3669 & 0.0961 \\
\hline & 3 D-Elasticity[16] & 0.5781 & 0.4295 & 0.0584 & 0.0689 & 0.3696 & 0.0981 \\
\hline \multirow{2}{*}{30} & Present & 0.4726 & 0.2605 & 0.1025 & 0.1252 & 0.2924 & 0.1723 \\
\hline & 3 D-Elasticity[16] & 0.5087 & 0.2662 & 0.1037 & 0.1290 & 0.2957 & 0.1749 \\
\hline \multirow{2}{*}{45} & Present & 0.4420 & 0.1596 & 0.1596 & 0.1487 & 0.2323 & 0.2323 \\
\hline & 3 D-Elasticity[16] & 0.4890 & 0.1640 & 0.1640 & 0.1548 & 0.2355 & 0.2355 \\
\hline
\end{tabular}


Higher Order Refined Computational Model using Post Processing for the Transverse Shear and Transverse Normal Stress in Composite Plates

\section{CONCLUSION}

The results obtained are in very good agreement with 3D elasticity solutions. Hence ESL theory with higher degrees of freedom with post processing technique can predict the local behavior i.e. transverse stresses accurately.

\section{REFERENCES}

[1] Reddy, J. N. (1993). An Evaluation of Equivalent-Single-Layer and Layerwise Theories of Composite Laminates, Composite Structures, 25: 21-35.

[2] Noor, A. K., Burton, W. S. and Bert, C. W. (1996). Computational Models for Sandwich Panels and Shells, Applied Mechanics Reviews, 49: 155-199.

[3] Kant, T. and Swaminathan, K. (2000) Estimation of Transverse/Interlaminar Stresses in Laminated Composites- A Selective Review and Survey of Current Developments, Composite Structures, 49: 65-75.

[4] Ghugal, Y. M. and Shimpi, R. P. (2002). A Review of Refined Shear Deformation Theories of Isotropic and Anisotropic Laminated Plates, J. Reinf. Plastics Composites, 21: 775-813.

[5] Zhang, Y. X. and Yang, C. H. (2009). Recent Developments in Finite Element Analysis For Laminated Composite Plates, Composite Structures, 88: 147-157.

[6] Atteshamuddin S. Sayyad, Yuwaraj M. Ghugal, On the free vibration analysis of laminated composite and sandwich plates: A review of recent literature with some numerical results, Composite Structures, 129 (2015) 177-201

[7] K.M. Liew et al., An overview of layerwise theories for composite laminates and structures: Development, numerical implementation and application, Composite Structures, 216 (2019) 240-259

[8] Hiroyuk Matsunaga (2002). Assessment of A Global Higher-Order Deformation Theory for Laminated Composite and Sandwich Plates, Composite Structures, 56: 279-291.

[9] Sa Sudha Ramesh et al. (2009), A higher-order plate element for accurate prediction of interlaminar stresses in laminated composite plates, Composite Structures, 91 (2009) 337-357

[10] P.H. Shah, R.C. Batra, (2015) Through-the-thickness stress distributions near edges of composite laminates using stress recovery scheme and third order shear and normal deformable theory, Composite Structures, 131, 397-413

[11] X. Wang, G. Sh (2015), A refined laminated plate theory accounting for the third-order shear deformation and interlaminar transverse stress continuity, Applied Mathematical Modelling, (2015) Accepted Manuscrpt

[12] Kant, T. and Manjunatha B. S. (1988). An Unsymmetric FRC Laminate C0 Finite Element Model with 12 Degrees of Freedom Per Node, Eng Compu, 5: 300-308.

[13] Swaminathan, K. and Patil S. S. (2007). Higher Order Refined Computational Model with 12 Degrees of Freedom for the Stress Analysis of Antisymmetric Angle-Ply Plates - Analytical Solutions, Composite Structures, 80: 595-608.

[14] Swaminathan, K. and Patil, S. S. (2008). Analytical Solutions Using a Higher Order Refined Computational Model with 12 Degrees of Freedom for the Free Vibration Analysis of Antisymmetric Angle-Ply Plates, Composite Structures, 82: 209-216. 
[15] Swaminathan, K., Patil, S. S., Nataraja, M. S. and Mahabaleswara, K. S. (2006). Bending of Sandwich Plates with Anti-Symmetric Angle-Ply Face Sheets - Analytical Evaluation of Higher Order Refined Computational Models, Composite Structures, 75: 114-120.

[16] Savoia, M. and Reddy, J. N. (1992). A variational approach to three-dimensional elasticity solutions of laminated composite plates. J. Appl. Mech. 59, 166-175.

[17] Reddy, J. N. (1996). Mechanics of Laminated Composite Plates, Theory and Analysis, Boca Raton, USA: CRC Press. 